\section{Do 2019 European League against rheumatism/ American College of Rheumatology classification criteria for systemic lupus erythematosus also indicate the disease activity?}

The 2019 European League against rheumatism/American College of Rheumatology classification criteria (2019 criteria) for systemic lupus erythematosus (SLE) has introduced a new scoring system to classify SLE. ${ }^{1}$ It is a thrill for rheumatologists to get the new SLE classification criteria, which has both excellent sensitivity and specificity, and further demonstrated by other studies to be effective in the early SLE diagnosis and distinguish patients with SLE from patients with primary Sjögren's syndrome. ${ }^{2}$ It has also been reported that higher scores of 2019 criteria were associated with higher rates of organ damage. ${ }^{4}$ While using antinuclear antibody (ANA) as an entry criterion, these hierarchically clustered and weighted criteria made a significant breakthrough compared with the past several criteria. However, based on the thinking of weighted criteria, we are curious as to whether they can reflect the disease activity of SLE? Because assessing tools such as SLE disease activity index (SLEDAI) and revised systemic lupus activity measure (SLAM-R) also use weighted scores widely, could these weighted items in the new criteria have the same trend?

Thus, we enrolled 96 consecutive and hospitalised new-onset SLE patients in the Department of Rheumatology and Immunology of Ruijin Hospital from August 2016 to June 2018. The data were collected through the electronic medical records. Two qualified senior rheumatologists (JT and CY) confirmed the diagnosis of SLE according to the 2019 criteria. SLEDAI and SLAM-R were recorded when the patients were hospitalised and confirmed by another two qualified rheumatologists (JY and ZZ). British Isles Lupus Assessment Group was not used in this study. Because it should be compared with the previous visit's disease activity, while, there is only one visit available for newonset patients. This study was approved by the Ethics Committee of Ruijin Hospital.

As a result, the mean age was $41 \pm 16$ years and the mean duration was $19 \pm 53$ months. Eighty-three (86\%) were female and $13(14 \%)$ were male. The detailed distribution of clinical characteristics according to 2019 criteria were shown in table 1. It was interesting to find out that 2019 criteria correlated positively with SLEDAI $\left(\mathrm{p}<0.0001, \mathrm{r}^{2}=0.65\right)$ and SLAM-R $\left(\mathrm{p}<0.0001, \mathrm{r}^{2}=0.37\right)$ (figure 1$)$. Compared with SLAM-R, SLEDAI were more convergent and correlated better with the scores of 2019 criteria. Both correlations provided
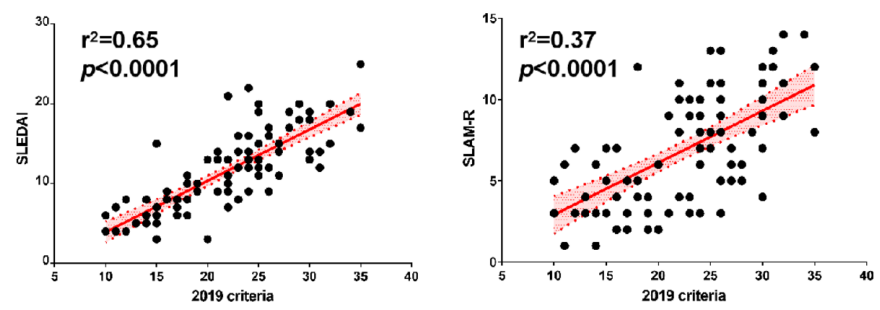

Figure 1 The correlation of 2019 criteria with SLEDAI and SLAM-R. 2019 criteria correlated positively with SLEDAI $\left(p<0.0001, r^{2}=0.65\right)$ and SLAM-R $\left(p<0.0001, r^{2}=0.37\right)$. SLAM- $R$, revised systemic lupus activity measure; SLEDAI, SLE disease activity index.
Table 1 The clinical characteristics of new-onset SLE patients diagnosed with 2019 criteria

\begin{tabular}{ll}
\hline Clinical criteria & Positive/total patients (\%) \\
\hline $\mathrm{N}$ & 96 \\
\hline Fever & $40 / 96(42)$ \\
\hline Cutaneous domain & \\
\hline Acute cutaneous lupus & $24 / 96(25)$ \\
\hline Subacute cutaneous lupus or discord rash & $25 / 96(26)$ \\
\hline Non-scaring alopecia & $19 / 96(20)$ \\
\hline Oral ulcer & $18 / 96(19)$ \\
\hline Arthritis domain & $51 / 96(53)$ \\
\hline Serositis domain & $25 / 96(26)$ \\
\hline Pleural or pericardial effusion & $21 / 96(22)$ \\
\hline Acute pericarditis & $4 / 96(4)$ \\
\hline Renal domain & $37 / 96(39)$ \\
\hline Protein >0.5 g/24hours & $37 / 96(39)$ \\
\hline Class II or V lupus nephritis & $0 / 96(0)$ \\
\hline Class III or IV lupus nephritis & $0 / 96(0)$ \\
\hline Neurologic domain & \\
\hline Seizure & $0 / 96(0)$ \\
\hline Psychosis & $0 / 96(0)$ \\
\hline Delirium & $0 / 96(0)$ \\
\hline Haematologic domain & \\
\hline Leucopenia & $61 / 96(64)$ \\
\hline Thrombocytopenia & $31 / 96(32)$ \\
\hline Autoimmune haemolysis & $57 / 96(59)$ \\
\hline Laboratory criteria & Positive/total patients (\%) \\
\hline Immunologic & $10 / 96(10)$ \\
\hline Anticardiolipin antibodies & $23 / 96(24)$ \\
\hline Antibeta2-glycoprotein antibodies & $26 / 96(27)$ \\
\hline Lupus anticoagulant & $86 / 96(90)$ \\
\hline Complement domain & $87 / 96(91)$ \\
\hline Anti-dsDNA anti-Sm antibody & $20 / 96(21)$ \\
\hline Anti-dsDNA, anty specific antibodies domain & \\
\hline
\end{tabular}

Anti-dsDNA, antibodies to double-stranded DNA; Anti-Sm, anti-Smith; SLE, systemic lupus erythematosus.

some clues that the score of 2019 criteria might also indicate the disease activity.

In our study, it is the first attempt to associate 2019 criteria with SLEDAI and SLAM-R. SLEDAI correlates better with 2019 criteria, partly because items in 2019 criteria are similar to those in SLEDAI. However, in 2019 criteria, with each domain, only the highest weighted criterion is counted, while, in SLEDAI, they are counted separately. ${ }^{15}$ SLAM-R has items that exist neither in 2019 criteria nor in SLEDAI, which may finally lead to the difference in the analysis of correlation.

Besides, when using 2019 criteria in the clinic, it increases burden for rheumatologists to calculate scores, and then to calculate SLEDAI or SLAM-R. Considering the efforts for the rheumatologists to memorise 2019 criteria and two more disease activity score systems, it raises a question whether it is possible in the future we will have new criteria that could be used both as disease activity and classifying criteria?

Jialin Teng $\odot$, Zhuochao Zhou, Fan Wang, Yue Sun, Hong-Lei Liu, Xiaobing Cheng, Yutong Su ${ }^{\circ}$, Hui Shi, Chengde Yang $\odot$, Junna Ye

Department of Rheumatology and Immunology, Ruijin Hospital,Shanghai Jiao Tong University School of Medicine, Shanghai, China

Correspondence to Junna Ye, Shanghai, China; yjn0912@qq.com 
Contributors Concept and writing: JY and JT; revising: YS and YD; acquisition of data: ZZ, FW and YS; analysis and interpretation of data: LL, XC and HS.

Funding This work is supported by National Natural Science Foundation of China (81801592).

Competing interests None declared.

Patient consent for publication Not required.

Ethics approval This study was approved by the Ethics Committee of Ruijin Hospital (ID:2016-62)

Provenance and peer review Not commissioned; internally peer reviewed.

(c) Author(s) (or their employer(s)) 2020. No commercial re-use. See rights and permissions. Published by BMJ.

\section{A) Check for updates}

To cite Teng J, Zhou Z, Wang F, et al. Ann Rheum Dis Epub ahead of print: [please include Day Month Year]. doi:10.1136/annrheumdis-2020-217017

Received 19 January 2020

Accepted 24 January 2020

\section{SLinked}

http://dx.doi.org/10.1136/annrheumdis-2020-217105
Ann Rheum Dis 2020;0:1-2. doi:10.1136/annrheumdis-2020-217017

\section{ORCID iDs}

Jialin Teng http://orcid.org/0000-0003-1016-9064

Yutong Su http://orcid.org/0000-0003-0488-2939

Chengde Yang http://orcid.org/0000-0002-3720-634X

\section{REFERENCES}

1 Aringer M, Costenbader K, Daikh D, et al. 2019 European Leaque against Rheumatism/ American College of rheumatology classification criteria for systemic lupus erythematosus. Ann Rheum Dis 2019;78:1151-9.

2 Adamichou C, Nikolopoulos D, Genitsaridi I, et al. In an early SLE cohort the ACR1997, SLICC-2012 and EULAR/ACR-2019 criteria classify non-overlapping groups of patients: use of all three criteria ensures optimal capture for clinical studies while their modification earlier classification and treatment. Ann Rheum Dis 2020;79:232-41.

3 Carneiro AC, Ruiz MM, Freitas S, et al. A comparison of three classification criteria sets for systemic lupus erythematosus - a study looking at links to outcome and mortality. Arthritis Care Res 2019. doi:10.1002/acr.24061. [Epub ahead of print: 10 Sep 2019].

4 Assan F, Seror R, Mariette X, et al. New 2019 SLE EULAR/ACR classification criteria are valuable for distinguishing patients with SLE from patients with pSS. Ann Rheum Dis 2019. doi:10.1136/annrheumdis-2019-216222. [Epub ahead of print: 9 Sep 2019].

5 Romero-Diaz J, Isenberg D, Ramsey-Goldman R. Measures of adult systemic lupus erythematosus: updated version of British Isles lupus assessment group (BILAG 2004), European consensus lupus activity measurements (ECLAM), systemic lupus activity measure, revised (SLAM-R), systemic lupus activity questionnaire for population studies (SLAQ), systemic lupus erythematosus disease activity index 2000 (SLEDAI-2K), and systemic lupus international collaborating Clinics/American College of rheumatology damage index (SDI). Arthritis Care Res 2011;63 Suppl 11:S37-46. 